\title{
Non-reciprocity of Faraday rotation in gyrotropic crystals
}

\author{
Vlokh R. and Adamenko D. \\ Institute of Physical Optics, 23 Dragomanov St., 79005 Lviv, Ukraine \\ e-mail: vlokh@ifo.lviv.ua
}

Received: 29.08.2008

\begin{abstract}
It is shown that, under the conditions of coexisting natural optical activity and nonzero linear optical birefringence, reversal of the light wave vector sign can result in changing angle of Faraday rotation.
\end{abstract}

Keywords: natural optical activity, Faraday rotation, magnetogyration, non-reciprocity

PACS: 33.55.Ad, 78.20.Ls, 78.20.Ek, 78.20.Fm

UDC: 535.37

\section{Introduction}

The effect of additional optical rotation observed earlier in a number of absorbing non-centrosymmetric crystals under the influence of external magnetic field [1-3], which has been explained as a magnetogyration (MG) [4-6], is usually distinguished from the Faraday rotation (FR) via reversing the wave vector of light or rotating a crystal sample by $180^{\circ}$ around the axis perpendicular to the optic axis of the crystal. In relation to this problem, it is worth noticing that the MG as a spatial dispersion effect induced by magnetic field should not, in principle, manifest itself as an optical rotation. Indeed, according to the known Onsager principle, it should lead to changes in the real part of the dielectric permittivity (or the refractive indices), as a result of the so-called ' $k H$-effect' [7]. The MG effect has been widely discussed in the last decades [8-11], though the common point of view has not yet been established. In our recent papers it has been shown that non-complete non-reciprocity of the Faraday rotation exists even in quartz crystals, in spite of the fact that the MG effect is forbidden by the point symmetry of those crystals $[12,13]$ for the experimental geometry used in the works [12, 13] $(k\|H\| Z)$. Thus, the effect observed has no relation with the MG phenomenon. Moreover, non-complete non-reciprocity of the Faraday rotation has been observed in crystals which possess natural optical activity (for comparison see [12-14]). In the present paper we will demonstrate how natural optical activity and non-zero linear optical birefringence can lead to the non-complete non-reciprocal Faraday rotation. 


\section{Theoretical analysis}

The relation for the electric field $E_{i}$ of optical wave with accounting for the spatial dispersion effects can be written as follows [15]:

$$
E_{i}=B_{i j} D_{j}+i \eta_{i j k} \frac{\partial D_{j}}{\partial x_{k}}=\left(B_{i j}+i \eta_{i j k} k_{k}\right) D_{j},
$$

where $D_{j}$ is the electric induction at the optical frequency, $B_{i j}$ optical-frequency impermeability tensor, $k_{k}$ the wave vector of light, $\eta_{i j k}$ a polar antisymmetric $\left(\eta_{i j k}=-\eta_{j i k}\right)$ third-rank tensor. Using the known duality relation and normalizing factor $\frac{2 \pi}{\lambda}$, one can rewrite the tensor $\eta_{i j k}$ as a second-rank axial tensor (the gyration tensor):

$$
g_{l k}=\frac{2 \pi}{\lambda} e_{l i j} \eta_{i j k},
$$

where $e_{l i j}$ denotes the unit completely antisymmetric Levi-Civita tensor and $\lambda$ the wavelength of optical radiation. If external magnetic field $H_{k}$ exists, a term should be included in Eq. (1) that describes the Faraday effect:

$$
E_{i}=\left(B_{i j}+i e_{i j k} g_{l k} m_{k}+i e_{j i k} F_{l k} H_{k}\right) D_{j}=\left[B_{i j}+i e_{i j k}\left(g_{l k} m_{k}+F_{l k} H_{k}\right)\right] D_{j},
$$

where $F_{l k}$ is the Faraday tensor. The wave vector $k$ can be written as

$$
k=\frac{2 \pi}{\lambda} m,
$$

with $m$ representing the unit wave-normal vector.

Let us now consider the relations for ellipticity of eigen waves and orientation of the azimuth of polarization ellipse for the material media with different properties. In the case of existence of only natural optical activity in a transparent optically uniaxial crystal and the conditions $m_{3} ; g_{33} ; B_{11}=B_{22} \neq B_{33}$ Eq. (3) can be rewritten in the following form:

$$
E_{i}=\left(B_{i j}+i e_{i j l} g_{l k} m_{k}\right) D_{j} .
$$

In the matrix representation this relation looks as follows:

\begin{tabular}{c|ccc} 
& $D_{1}$ & $D_{2}$ & $D_{3}$ \\
\hline$E_{1}$ & $B_{11}$ & $i g_{33} m_{3}$ & 0 \\
$E_{2}$ & $-i g_{33} m_{3}$ & $B_{11}$ & 0 \\
$E_{3}$ & 0 & 0 & $B_{33}$
\end{tabular}.

This can also be written as a system of equations: 


$$
\left\{\begin{array}{c}
E_{1}=B_{11} D_{1}+i g_{33} m_{3} D_{2} \\
E_{2}=-i g_{33} m_{3} D_{1}+B_{11} D_{2}
\end{array} .\right.
$$

Taking into account the relation $B_{11}=\left(\frac{1}{n_{0}^{2}}\right)$ (with $n_{0}$ being the refractive index not influenced by the optical activity), one can get

$$
\left\{\begin{array}{c}
E_{1}=\left(\frac{1}{n_{0}^{2}}\right) D_{1}+i g_{33} m_{3} D_{2} \\
E_{2}=-i g_{33} m_{3} D_{1}+\left(\frac{1}{n_{0}^{2}}\right) D_{2}
\end{array} .\right.
$$

Let us use the relations $E_{1}=\frac{D_{1}}{n^{2}}, E_{2}=\frac{D_{2}}{n^{2}}$. Then the system of Eqs. (8) may be represented as

$$
\left\{\begin{array}{l}
\left(\frac{1}{n_{0}^{2}}-\frac{1}{n^{2}}\right) D_{1}+i g_{33} m_{3} D_{2}=0 \\
-i g_{33} m_{3} D_{1}+\left(\frac{1}{n_{0}^{2}}-\frac{1}{n^{2}}\right) D_{2}=0
\end{array}\right.
$$

As one can easily see, the system of Eqs. (9) can be reduced to a quadratic equation with the unknown $n$ :

$$
\left(\frac{1}{n_{0}^{2}}-\frac{1}{n^{2}}\right)^{2}-\left(g_{33} m_{3}\right)^{2}=0
$$

or

$$
\left(\frac{1}{n_{0}^{2}}\right)^{2}-2\left(\frac{1}{n_{0}^{2}}\right)\left(\frac{1}{n^{2}}\right)+\left(\frac{1}{n^{2}}\right)^{2}-\left(g_{33} m_{3}\right)^{2}=0,
$$

with the solutions

$$
\frac{1}{n_{0}^{2}}-\frac{1}{n^{2}}= \pm g_{33} m_{3}, \quad \frac{1}{n^{2}}=\frac{1}{n_{0}^{2}} \mp g_{33} m_{3}
$$

Thus, the refractive indices for the right and left circularly polarized waves are as follows:

$$
n_{r, l}=\frac{1}{\sqrt{\frac{1}{n_{0}^{2}} \mp g_{33} m_{3}}} \approx n_{0} \pm \frac{1}{2} n_{0}^{3} g_{33} m_{3},
$$

while the circular birefringence is determined by the relation 


$$
\Delta n_{C}=n_{r}-n_{l}=n_{0}^{3} g_{33} m_{3} .
$$

The ellipticity of the eigen light waves can be determined from the relations $\frac{D_{1}}{D_{2}}=-i \kappa$ and $\frac{D_{2}}{D_{1}}=i \kappa$. In the case described above these are equal to \pm 1 and so correspond to the right and left circular waves.

The optical retardation $\Delta$ which appears when the two circularly polarized waves traverse the distance $d$ in the crystal is defined by the relation

$$
\Delta=\frac{2 \pi d}{\lambda} n_{0}^{3} g_{33} m_{3}
$$

while the angle of rotation of the polarization plane is given by

$$
\varphi=\frac{1}{2} \Delta=\frac{\pi d}{\lambda} n_{0}^{3} g_{33} m_{3} .
$$

Hence, in the conditions if only the natural optical activity exists, the angle of rotation of the polarization plane would change its $\operatorname{sign}\left(\varphi^{\left(m_{3}\right)} \rightarrow-\varphi^{\left(-m_{3}\right)}\right)$ under reversal of the wave vector $\left(m_{3} \rightarrow-m_{3}\right)$.

Let now both the Faraday rotation and the natural optical activity coexist in a transparent optically uniaxial crystal $\left(m_{3} ; g_{33} ; F_{33} ; B_{11}=B_{22} \neq B_{33}\right)$. Then one should consider Eq. (3), which in the matrix representation can be written as follows:

\begin{tabular}{c|ccc} 
& $D_{1}$ & $D_{2}$ & $D_{3}$ \\
\hline$E_{1}$ & $B_{11}$ & $i\left(g_{33} m_{3}+F_{33} H_{3}\right)$ & 0 \\
$E_{2}$ & $-i\left(g_{33} m_{3}+F_{33} H_{3}\right)$ & $B_{11}$ & 0 \\
$E_{3}$ & 0 & 0 & $B_{33}$
\end{tabular}.

Similar to the previous case, solving the system of equations

$$
\left\{\begin{array}{c}
E_{1}=B_{11} D_{1}+i\left(g_{33} m_{3}+F_{33} H_{3}\right) D_{2} \\
E_{2}=-i\left(g_{33} m_{3}+F_{33} H_{3}\right) D_{1}+B_{11} D_{2}
\end{array},\right.
$$

or

$$
\left\{\begin{array}{c}
E_{1}=\left(\frac{1}{n_{0}^{2}}\right) D_{1}+i\left(g_{33} m_{3}+F_{33} H_{3}\right) D_{2} \\
E_{2}=-i\left(g_{33} m_{3}+F_{33} H_{3}\right) D_{1}+\left(\frac{1}{n_{0}^{2}}\right) D_{2}
\end{array}\right.
$$

with taking the relations $E_{1}=\frac{D_{1}}{n^{2}}$ and $E_{2}=\frac{D_{2}}{n^{2}}$ into account, 


$$
\left\{\begin{array}{l}
\left(\frac{1}{n_{0}^{2}}-\frac{1}{n^{2}}\right) D_{1}+i\left(g_{33} m_{3}+F_{33} H_{3}\right) D_{2}=0 \\
-i\left(g_{33} m_{3}+F_{33} H_{3}\right) D_{1}+\left(\frac{1}{n_{0}^{2}}-\frac{1}{n^{2}}\right) D_{2}=0
\end{array},\right.
$$

one can arrive at the quadratic equation

$$
\left(\frac{1}{n_{0}^{2}}-\frac{1}{n^{2}}\right)^{2}-\left(g_{33} m_{3}+F_{33} H_{3}\right)^{2}=0 .
$$

Its solutions for the refractive indices of the right and left waves are given by

$$
n_{r, l}=\frac{1}{\sqrt{\frac{1}{n_{0}^{2}} \mp\left(g_{33} m_{3}+F_{33} H_{3}\right)}} \approx n_{0} \pm \frac{1}{2} n_{0}^{3}\left(g_{33} m_{3}+F_{33} H_{3}\right) \text {. }
$$

From the relations $\frac{D_{1}}{D_{2}}=-i \kappa$ and $\frac{D_{2}}{D_{1}}=i \kappa$ it follows that the eigen waves should be again circularly polarized, with the ellipticities $\kappa= \pm 1$.

The circular birefringence, the optical retardation and the angle of rotation of the polarization plane are respectively equal to

$$
\begin{aligned}
& \Delta n_{C}=n_{r}-n_{l}=n_{0}^{3}\left(g_{33} m_{3}+F_{33} H_{3}\right), \\
& \Delta=\frac{2 \pi d}{\lambda} n_{0}^{3}\left(g_{33} m_{3}+F_{33} H_{3}\right), \\
& \varphi=\frac{1}{2} \Delta=\frac{\pi d}{\lambda} n_{0}^{3}\left(g_{33} m_{3}+F_{33} H_{3}\right) .
\end{aligned}
$$

As one can see from Eqs. (25), alteration of the light propagation direction to the opposite one $\left(m_{3} \rightarrow-m_{3}\right)$ leads to a difference of the corresponding rotation angles of the polarization plane. Moreover, these angles differ by both their signs and magnitudes:

$$
\varphi^{\left(m_{3}\right)}=\frac{1}{2} \Delta=\frac{\pi d}{\lambda} n_{0}^{3}\left(g_{33} m_{3}+F_{33} H_{3}\right), \quad \varphi^{\left(-m_{3}\right)}=\frac{1}{2} \Delta=\frac{\pi d}{\lambda} n_{0}^{3}\left(-g_{33} m_{3}+F_{33} H_{3}\right)
$$

Thus, we have $|\varphi|^{\left(m_{3}\right)} \neq|\varphi|^{\left(-m_{3}\right)}$. Such the difference follows from different behaviours of the natural optical activity and the Faraday rotation under reversing wave vector: the optical activity is then compensated, while the Faraday rotation becomes doubled.

Finally, let us consider the case when the natural optical activity, the Faraday rotation and some non-zero linear optical birefringence coexist in a transparent optically (quasi-) uniaxial crystal. We have $m_{3} ; g_{33} ; F_{33} ; B_{11} \neq B_{22} \neq B_{33}$ and the matrix given by Eq. (17) should be rewritten as 


\begin{tabular}{c|ccc} 
& $D_{1}$ & $D_{2}$ & $D_{3}$ \\
\hline$E_{1}$ & $B_{11}$ & $i\left(g_{33} m_{3}+F_{33} H_{3}\right)$ & 0 \\
$E_{2}$ & $-i\left(g_{33} m_{3}+F_{33} H_{3}\right)$ & $B_{22}$ & 0 \\
$E_{3}$ & 0 & 0 & $B_{33}$
\end{tabular}.

Then the system of equations

$$
\left\{\begin{array}{c}
E_{1}=B_{11} D_{1}+i\left(g_{33} m_{3}+F_{33} H_{3}\right) D_{2} \\
E_{2}=-i\left(g_{33} m_{3}+F_{33} H_{3}\right) D_{1}+B_{22} D_{2}
\end{array},\right.
$$

which can be finally rewritten as

$$
\left\{\begin{array}{l}
\left(\frac{1}{n_{01}^{2}}-\frac{1}{n^{2}}\right) D_{1}+i\left(g_{33} m_{3}+F_{33} H_{3}\right) D_{2}=0 \\
-i\left(g_{33} m_{3}+F_{33} H_{3}\right) D_{1}+\left(\frac{1}{n_{02}^{2}}-\frac{1}{n^{2}}\right) D_{2}=0
\end{array},\right.
$$

leads to the following quadratic equations:

$$
\left(\frac{1}{n_{01}^{2}}-\frac{1}{n^{2}}\right)\left(\frac{1}{n_{02}^{2}}-\frac{1}{n^{2}}\right)-\left(g_{33} m_{3}+F_{33} H_{3}\right)^{2}=0,
$$

or

$$
\frac{1}{n_{01}^{2} n_{02}^{2}}-\frac{1}{n_{01}^{2} n^{2}}-\frac{1}{n_{02}^{2} n^{2}}+\frac{1}{n^{4}}-\left(g_{33} m_{3}+F_{33} H_{3}\right)^{2}=0
$$

where $n_{01}$ and $n_{02}$ are the refractive indices that disregard the influence of the optical activity. Introducing the notation $\frac{1}{n^{2}}=x$, one can represent Eq. (31) as follows:

$$
x^{2}-\left(\frac{1}{n_{01}^{2}}+\frac{1}{n_{02}^{2}}\right) x+\frac{1}{n_{01}^{2} n_{02}^{2}}-\left(g_{33} m_{3}+F_{33} H_{3}\right)^{2}=0 .
$$

Its solutions are given by the relations

$$
\begin{aligned}
& x=\frac{1}{2}\left[\left(\frac{1}{n_{01}^{2}}+\frac{1}{n_{02}^{2}}\right) \pm \sqrt{\left(\frac{1}{n_{01}^{2}}+\frac{1}{n_{02}^{2}}\right)^{2}-4\left(\frac{1}{n_{01}^{2} n_{02}^{2}}-\left(g_{33} m_{3}+F_{33} H_{3}\right)^{2}\right)}\right]= \\
& =\frac{1}{2}\left[\left(\frac{1}{n_{01}^{2}}+\frac{1}{n_{02}^{2}}\right) \pm \sqrt{\left(\frac{1}{n_{01}^{2}}-\frac{1}{n_{02}^{2}}\right)^{2}+4\left(g_{33} m_{3}+F_{33} H_{3}\right)^{2}}\right]= \\
& =\frac{1}{2}\left[\left(\frac{1}{n_{01}^{2}}+\frac{1}{n_{02}^{2}}\right) \pm \sqrt{\left(\frac{1}{n_{01}^{2}}-\frac{1}{n_{02}^{2}}\right)^{2}+4\left(g_{33} m_{3}\right)^{2}+4\left(F_{33} H_{3}\right)^{2}+8 g_{33} F_{33} H_{3} m_{3}}\right] .
\end{aligned}
$$


Let us make use of the notation $\Xi=g_{33} m_{3}+F_{33} H_{3}$. Then the ellipticity of the eigen waves can be represented as

$$
\begin{aligned}
& \kappa=\frac{\Xi}{\frac{1}{n_{02}^{2}}-\frac{1}{n^{2}}}=\frac{\Xi}{\frac{1}{n_{02}^{2}}-\frac{1}{2 n_{01}^{2}}-\frac{1}{2 n_{02}^{2}} \mp \frac{1}{2} \sqrt{\left(\frac{1}{n_{01}^{2}}-\frac{1}{n_{02}^{2}}\right)^{2}+4 \Xi^{2}}}= \\
& =\frac{\Xi}{\frac{1}{2}\left[\left(\frac{1}{n_{02}^{2}}-\frac{1}{n_{01}^{2}}\right) \mp \sqrt{\left(\frac{1}{n_{01}^{2}}-\frac{1}{n_{02}^{2}}\right)^{2}+4 \Xi^{2}}\right]}||_{n_{02} \geq n_{01}}= \\
& =\frac{g_{33} m_{3}+F_{33} H_{3}}{\bar{n}^{3} \mp \frac{1}{2} \sqrt{\left(\frac{\Delta n}{\bar{n}^{3}}\right)^{2}+4\left(\left(g_{33}\right)^{2}+2 g_{33} F_{33} H_{3} m_{3}+\left(F_{33} H_{3}\right)^{2}\right)}},
\end{aligned}
$$

where $\Delta n$ and $\bar{n}$ are the optical birefringence and the mean refractive index, respectively.

As one can see, the ellipticity of the eigen waves is not equal to unity, i.e. the eigen waves are elliptically polarized, the electric field vectors of each of them being right- or left-handed. The refractive indices of those elliptically polarized waves $n_{r, l}$, the elliptical birefringence $\Delta n_{r, l}$ and the optical retardation are defined respectively by the relations

$$
\begin{aligned}
& n_{r, l}=\frac{1}{\sqrt{\frac{1}{n^{2}} \pm \sqrt{\left(\frac{\Delta n}{\bar{n}^{3}}\right)^{2}+\Xi^{2}}} \approx} \\
& \approx n \mp \frac{1}{2} n^{3} \sqrt{\left(\frac{\Delta n}{\bar{n}^{3}}\right)^{2}+\left(g_{33}\right)^{2}+2 g_{33} F_{33} H_{3} m_{3}+\left(F_{33} H_{3}\right)^{2}}, \\
& \Delta n_{r, l}=n^{3} \sqrt{\left(\frac{\Delta n}{\bar{n}^{3}}\right)^{2}+\left(g_{33}\right)^{2}+2 g_{33} F_{33} H_{3} m_{3}+\left(F_{33} H_{3}\right)^{2}}, \\
& \Delta=\frac{2 \pi d}{\lambda} n^{3} \sqrt{\left(\frac{\Delta n}{\bar{n}^{3}}\right)^{2}+\left(g_{33}\right)^{2}+2 g_{33} F_{33} H_{3} m_{3}+\left(F_{33} H_{3}\right)^{2}} .
\end{aligned}
$$

Let us remind that for the incident azimuths $\alpha=0$ the relation for the rotation of the polarization ellipse includes both the eigen wave ellipticity and the optical retardation: 


$$
\tan 2 \varphi=-\frac{\frac{2 \kappa}{1+\kappa^{2}} \sin \Delta}{\left(\frac{1-\kappa^{2}}{1+\kappa^{2}}\right)^{2}+\left(\frac{2 \kappa}{1+\kappa^{2}}\right)^{2} \cos \Delta}
$$

Hence, the additional term $\left(2 g_{33} F_{33} H_{3} m_{3}\right)$ is available in Eqs. (34) and (37), which finally define the rotation angle of the light polarization ellipse. As a result, this angle should depend on both the magnetic field and the sign of the light wave vector. Actually, this term is a one that combines the natural optical activity and the Faraday effect. It appears owing to existence of a non-zero linear birefringence. Under the reversal of the wave vector, the corresponding elliptical retardations and the ellipticities of the eigen waves will differ due to existence of the term mentioned above. Then the rotation angles of the polarization ellipse would differ, too. In such a case non-complete non-reciprocity of the Faraday rotation not related to the MG effect would appear.

Hence, we deal here with an effect associated with superposition of circular optical birefringence (due to the natural optical activity and the Faraday rotation) and a linear birefringence. Such a superposition is known to may lead to some effects impossible for observing in a plane case of pure circular birefringence. A relevant example may be the influence of periodic structural modulation in crystals on their optical anisotropy characteristics, which cannot be reduced to that for the case of light propagation strictly along an optic axis direction [16]. Still closer to our subject is another example related to multiple light reflections in anisotropic dielectric media [17]: contrary to the case of the Faraday rotation, those reflections do not affect the optical rotation due to pure optical activity, though the effect appears whenever a small accompanying linear birefringence exists.

\section{Conclusions}

In the present work we have shown that, under the conditions of coexistence of the natural optical activity, the Faraday effect and some non-zero linear optical birefringence, a non-complete non-reciprocity of the Faraday rotation can appear in transparent, optically quasi-uniaxial crystals. In particular, such the conditions may be reached in optically uniaxial crystals that manifest some residual linear birefringence along the optic axis direction.

\section{References}

1. Zheludev I S, Vlokh O G and Sergatyuk V A, 1985. Magnetogyration and other phenomena described by second-rank axial tensors. Ferroelectrics. 63: 97-105.

2. Vlokh O G and Sergatyook V A, 1986. Magneto-polarization pseudo-gyration. Dokl. AN USSR. 291: 832-35.

3. Vlokh O G and Sergatyook V A, 1988. Pseudogyration effects in the lead germanate type ferroelectrics. Ferroelectrics. 80: 313-316.

4. Vlokh R, Vlokh O G, Klymiv I and Adamenko D, 2002. Magnetogyration in the CdS 
and $\left(\mathrm{Ga}_{\mathrm{x}} \operatorname{In}_{1-\mathrm{x}}\right)_{2} \mathrm{Se}_{3}$ crystals in the spectral range of interband absorption. Ukr. J. Phys. Opt. 3 271-76.

5. Vlokh R, Vlokh O G, Klymiv I and Adamenko D, 2002. Once more about magnetogyration (case of the CdS, $\left(\mathrm{Ga}_{\mathrm{x}} \mathrm{In}_{1-\mathrm{x}}\right)_{2} \mathrm{Se}_{3}, \mathrm{SiO}_{2}$ and $\mathrm{Li}_{2} \mathrm{~B}_{4} \mathrm{O}_{7}$ crystals). Ukr. J. Phys. Opt. 3: 166-170.

6. Vlokh O G and Vlokh R O, 1990. On the interpretation of the nontrivial optical activity in magnetic field. Opt. Spektr. 69: 458-459.

7. Krichevtsov B B, Pisarev R V , Rzhevsky A A, Gridnev V N and Weber H-J, 1998. Magnetospatial dispersion effect in magnetic semiconductors $\mathrm{Cd}_{1-x} \mathrm{Mn}_{x} \mathrm{Te}$. Phys. Rev. B57: 14611-14.

8. Zheludev N I, Popov S V, Svirko Yu P and Malinowski A, 1994. Observation of time-nonreversible optical interaction with zinc-blende semiconductors. Phys. Rev. B50: $11508-11513$.

9. Lew Yan Voon L C, Fainstein A, Etchegoin P, Santos P and Cardona M, 1995. Comment on "Observation of time-nonreversible optical interaction with zincblende semiconductors'”. Phys. Rev. B52: 2201-2202.

10. Zheludev N I, Popov S V, Svirko Yu P, Malinowski A and Bungay A R, 1995. Reply to "Comment on "Observation of time-nonreversible optical interaction with zinc-blende semiconductors”. Phys. Rev. B52: 2203-2205.

11. Vlokh O G and Vlokh R O, 1989. Symmetry aspects of the optical activity effects. Opt. Spektr. 69: 225-227.

12. Krupych O, Vasylkiv Yu, Adamenko D, Vlokh R and Vlokh O, 2006. Application of Small-Angular Magnetooptic Polarimetry for Study of Magnetogyration in $\left(\mathrm{Ga}_{0.3} \mathrm{In}_{0.7}\right)_{2} \mathrm{Se}_{3}$ and $\mathrm{SiO}_{2}$ Crystals. Ukr. J. Phys. Opt. 7: 171-178.

13. Vlokh R, Vasylkiv Yu, Adamenko D, Krupych O and Vlokh O G, 2008. Application of small-angular magnetooptic polarimetry for the study of magneto-gyration in $\left(\mathrm{Ga}_{0.3} \mathrm{In}_{0.7}\right)_{2} \mathrm{Se}_{3}, \mathrm{Bi}_{12} \mathrm{GeO}_{20}$ and $\mathrm{SiO}_{2}$ crystals. J. Opt. A: Pure Appl. Opt. 10: 015004 (9 pp).

14. Vlokh R O, Adamenko D I, Krupych O M and Vlokh O G, 2007. Small-Angular Polarimetric Magnetooptical Mapping and the Problem of Magnetogyration. Ferroelectrics. 348: 48-57.

15. Sirotin Yu I and Shaskolskaya M P, Fundamentals of crystal physics. Moscow: Nauka (1979).

16. Kushnir O S, 1997. Crystal optical properties of incommensurate phases in the plane-wave modulation region. J. Phys.: Condens. Matter. 9: 9259-9273.

17. Kushnir O S, 2004. Multiple reflections in crystals: natural and Faraday optical activity. Ukr. J. Phys. Opt. 5: 87-95. 